\title{
Quasinormal modes of a strongly coupled nonconformal plasma and approach to criticality
}

\author{
Panagiotis Betzios* \\ Institute for Theoretical Physics and Center for Extreme Matter and Emergent Phenomena, \\ Utrecht University, Princetonplein 5, 3584 CC Utrecht, Netherlands \\ Umut Gürsoy ${ }^{\dagger}$ \\ Institute for Theoretical Physics and Center for Extreme Matter and Emergent Phenomena, \\ Utrecht University, Leuvenlaan 4, 3584 CE Utrecht, Netherlands
}

Matti Järvinen

Institut de Physique Théorique Philippe Meyer and Laboratoire de Physique Théorique, École Normale Supérieure, PSL Research University, CNRS, 24 rue Lhomond, 75005 Paris, France

\section{Giuseppe Policastro ${ }^{\S}$}

Laboratoire de physique théorique, Département de physique de l' ENS, École normale supérieure PSL Research University, Sorbonne Universités, UPMC Univ. Paris 06, CNRS, 75005 Paris, France

(Received 1 December 2017; published 17 April 2018)

\begin{abstract}
We study fluctuations around equilibrium in a class of strongly interacting nonconformal plasmas using holographic techniques. In particular, we calculate the quasinormal mode spectrum of black hole backgrounds that approach Chamblin-Reall plasmas in the IR. In a specific limit, related to the exact linear-dilaton background in string theory, we observe that the plasma approaches criticality and we obtain the quasinormal spectrum analytically. We regulate the critical limit by gluing the IR geometry that corresponds to the nonconformal plasma to a part of AdS space-time in the UV. Near criticality, the spectrum can still be computed analytically and we find two sets of quasinormal modes, related to the IR and UV parts of the geometry. In the critical limit, the quasinormal modes accumulate to form a branch cut in the correlators of the energy-momentum tensor on the real axis of the complex frequency plane.
\end{abstract}

DOI: 10.1103/PhysRevD.97.081901

\section{INTRODUCTION}

Many applications of holographic duality concern systems around a critical point, where there is scale invariance. However, many realistic systems are not scale invariant in the regime of interest, QCD being a prominent example. It is then very interesting to study how the absence of scale invariance influences the transport properties of such systems in a controlled manner.

A simple class of models that allows this investigation is Einstein gravity coupled to a dilaton with an exponential

\footnotetext{
*P.Betzios@uu.nl

†U.Gursoy@uu.nl

*m.o.jarvinen@uu.nl

\$policast@lpt.ens.fr
}

Published by the American Physical Society under the terms of the Creative Commons Attribution 4.0 International license. Further distribution of this work must maintain attribution to the author(s) and the published article's title, journal citation, and DOI. Funded by SCOAP . potential $\propto e^{\alpha \phi}$, for which analytical black hole solutions are known [1]. We call the corresponding finite temperature system the Chamblin-Reall (CR) plasma. The parameter $\alpha$ determines the beta function of the dual theory and the deviation from conformality. At $\alpha=0$ we recover the conformal theory. This form of the potential is a good approximation for the IR behavior of a class of QCD-like holographic models [2,3].

In a previous paper [4] we studied time-dependent solutions of this model corresponding to boost-invariant flow, and found that the system has a slower approach to equilibrium than in the conformal case. In this article we continue this investigation, focusing on the quasinormal modes that contain information about the thermalization processes that lead to equilibrium. We are interested in the behavior of the modes as a function of the parameter $\alpha$. For $\alpha=0$ the spectrum is well known [5]: the modes lie along straight lines in the lower complex frequency half-plane.

We notice that there is a critical value $\alpha_{c}=4 / 3$ for which the string frame geometry matches with the twodimensional dilatonic black hole geometry, the linear 
dilaton background (plus some extra flat coordinates). The change in the geometry signals a phase transition, that can be shown to be continuous, of the BKT type $[6,7]$.

We have computed the spin-2 quasinormal spectrum analytically in the vicinity of the phase transition $\left(\alpha \rightarrow \alpha_{c}\right)$. We will present the main findings in this article; the details of the derivation and a more extensive presentation of the results will be given elsewhere [8]. We concentrate here on the results at zero momentum which actually apply to all fluctuations of the model, not just spin-2. In summary, we see that when we approach the critical point, the quasinormal modes become closely spaced and their imaginary part decreases, until eventually they form a branch cut on the real axis, even as the temperature remains finite at the critical point. We argue that this behavior signals a breakdown of hydrodynamics, as there are modes that decay more slowly than the hydrodynamic fluctuations. However, it is not clear if there is a dual theory exactly at the critical point (it has been suggested that it could correspond to an order-disorder transition in a spin model [9]).

We can have a well-defined dual theory if we consider a completion obtained by replacing part of the UV geometry with a slice of AdS spacetime. Even after the gluing, the whole spectrum of spin- 2 modes can be described analytically. The gluing introduces a new scale, and even at the critical point the modes are discrete; we observe that there are two distinct sets of modes with different behavior. There is a set which includes the lowest-lying modes, that are related to those of the CR geometry; these modes approach the modes of the pure CR geometry as the temperature is lowered (or equivalently the scale of the UV completion is increased). There is also another set of modes, starting at a higher frequency whose imaginary part is only mildly frequency-dependent, which we associate with the slice of the AdS space.

\section{QUASINORMAL MODES OF THE CR PLASMA}

We consider five dimensional Einstein-dilaton gravity

$$
\mathcal{A}=\frac{1}{16 \pi G_{5}} \int d^{5} x \sqrt{-g}\left(R-\frac{4}{3}(\partial \phi)^{2}+V_{0} e^{\alpha \phi}\right) .
$$

Defining

$$
f(\hat{r})=1-\left(\frac{\hat{r}}{\hat{r}_{h}}\right)^{\xi}, \quad \xi=\frac{4\left(1-9 \alpha^{2} / 64\right)}{1-9 \alpha^{2} / 16},
$$

so that $\xi \rightarrow \infty$ at the critical point $\alpha_{c}=4 / 3$, the exact CR solution of the model is given in the Eddington-Finkelstein coordinates as

$$
\begin{aligned}
d s^{2} & =e^{2 A_{0}} \hat{r}^{-\frac{2}{3}(\xi-1)}\left[-2 \ell^{\prime} d \hat{r} d v-f(\hat{r}) d v^{2}+\delta_{i j} d x^{i} d x^{j}\right] \\
\phi & =\frac{1}{2} \sqrt{(\xi-1)(\xi-4)} \log \hat{r}
\end{aligned}
$$

where $\ell^{\prime}=e^{-A_{0}} \xi \sqrt{1-1 / \xi} / \sqrt{V_{0}}$, and $A_{0}$ is a constant. For this geometry, the temperature reads

$$
T=\frac{\xi}{4 \pi \hat{r}_{h} \ell^{\prime}} .
$$

We now consider the spin-two fluctuations of the metric with momentum $k$ along the $x^{1}$-direction (the excitations being transverse to $k$ ). These can be shown to satisfy the relatively simple equation

$$
\begin{gathered}
\hat{r} f(\hat{r}) \Xi^{\prime \prime}(\hat{r})+\left(2 i \ell^{\prime} \hat{r} \omega+f(\hat{r})-\xi\right) \Xi^{\prime}(\hat{r}) \\
-\left(\ell^{\prime 2} k^{2} \hat{r}+(\xi-1) \ell^{\prime} i \omega\right) \Xi(\hat{r})=0 .
\end{gathered}
$$

All other fluctuations of the system also satisfy this same equation in the limit $k \rightarrow 0$.

Since the linear dilaton model is exactly solvable, we can find analytical results for the location quasinormal modes in a perturbation expansion in $\xi^{-1} \sim \alpha-\alpha_{c}$, which are corroborated by the numerical results. In particular, we can compute explicitly the analytic result for the correlator of the transverse components of the energy-momentum tensor to leading order in $1 / \xi$. In order to write down our result for the correlator, we define the reflection amplitude

$$
\mathcal{R}(\varpi, q)=-\frac{\Gamma(1+i \tilde{S}) \Gamma\left(\frac{1}{2}(1-i \varpi-i \tilde{S})\right)^{2}}{\Gamma(1-i \tilde{S}) \Gamma\left(\frac{1}{2}(1-i \varpi+i \tilde{S})\right)^{2}},
$$

where

$$
\tilde{S}=\sqrt{\varpi^{2}-q^{2}-1}
$$

with $\quad \varpi=\omega /(2 \pi T), \quad q=k /(2 \pi T)$. This amplitude describes the reflection of waves in the deep IR region of the geometry and is the same as in the minisuperspace studies of the two-dimensional black hole of the linear dilaton model $[10,11]$. The final result for the correlator is, however, more complicated due to a nontrivial interplay of the fluctuations in the UV and in the IR. In terms of the reflection amplitude the leading order result reads [8]

$$
G_{\mathrm{reg}}=\frac{2 \pi \xi \xi \hat{r}_{h}^{-\xi}}{\Gamma\left(\frac{\xi}{2}\right) \Gamma\left(1+\frac{\xi}{2}\right)}\left(\frac{\left(\varpi^{2}-q^{2}\right)}{16}\right)^{\frac{\xi}{2}}\left[i+\left(\frac{1+i \tilde{S}}{1-i \tilde{S}}\right)^{\frac{\xi}{2}} \frac{e^{-i \xi \tilde{S}}}{\mathcal{R}}\right]^{-1}
$$

for [12] $\operatorname{Re} \varpi \gtrsim \sqrt{1+q^{2}}$, and 


$$
\begin{aligned}
G_{\mathrm{reg}}= & \frac{2 \pi \xi^{\xi} \hat{r}_{h}^{-\xi}}{\Gamma\left(\frac{\xi}{2}\right) \Gamma\left(1+\frac{\xi}{2}\right)}\left(\frac{\left(\varpi^{2}-q^{2}\right)}{16}\right)^{\frac{\xi}{2}} \\
& \times\left[\left(\frac{1+S}{1-S}\right)^{\frac{\xi}{2}} e^{-\xi S} \mathcal{R}-i \theta(-\operatorname{Im} \varpi)\right]
\end{aligned}
$$

for $\operatorname{Re} \varpi \lesssim \sqrt{1+q^{2}}$. Here the subscript "reg" indicates that we have subtracted a singular term which does not contain any information on the quasinormal modes. We chose the branches of the square root factors such that the solution for negative $\varpi^{2}-q^{2}-1$ is given by replacing $\tilde{S} \mapsto i S$ where

$$
S=\sqrt{q^{2}-\varpi^{2}+1},
$$

which corresponds to analytic continuation through the upper half of the complex $\varpi$-plane.

The former expression (8) has a series of poles corresponding to the nonhydrodynamic quasinormal modes of the system. The main result is that the quasinormal modes become closely spaced and approach the real axis as $\xi \rightarrow \infty$. In the limit, they should create a branch cut on the real axis running from $\varpi=\sqrt{1+q^{2}}$ to $\varpi=\infty$ (see [13] for a recent discussion in a different context). This can be seen for the four lowest modes in Fig. 1. Notice that the branch cut is gapped; i.e., it starts from a frequency strictly larger than zero for any value of $q$.

The hydrodynamic quasinormal modes by contrast do not scale with $\xi$ : the imaginary part is fixed by the shear viscosity/entropy ratio that is $1 / 4 \pi$ independently of $\xi$. This means that one can find infinitely many nonhydro modes that are decaying slower than the hydro ones; which may suggest a break down of hydrodynamics in the limit $\xi \rightarrow \infty$.

\section{UV COMPLETION}

However, the critical limit is subtle. It is not possible to send $\xi \rightarrow \infty$ in Eq. (8) because of wildly oscillating phases

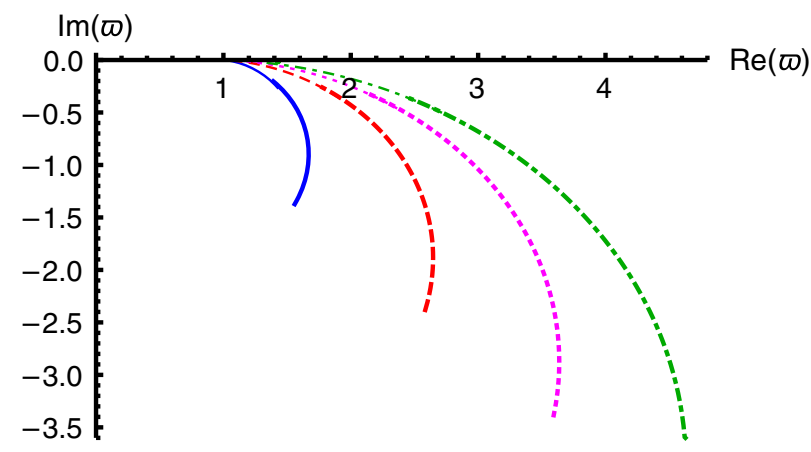

FIG. 1. The dependence of the four lowest nonhydrodynamic quasinormal modes on $\xi$ at $q=0$. Thick lines were obtained by directly solving the fluctuation equation (5) numerically for $4 \leq \xi \lesssim 20$, and the thin lines are based on the analytic approximation in (8). in the denominator. Thus at the critical point we do not have a well-defined prescription for computing correlators. This can be understood as a consequence of the fact that the geometry becomes asymptotically flat in the presence of a linear dilaton; the asymptotic behavior of the fluctuations allows instead to define a scattering S-matrix corresponding to the reflection amplitude (6). The definition of the boundary theory requires a UV regulator and the geometry being asymptotically AdS. For this reason, and also because the CR plasma is anyway only supposed to be an accurate description in the IR, we turn to the question of the robustness of our result when the theory is embedded into a UV complete description.

In order to implement this completion, we should consider dilaton potentials which admit a flow from AdS geometry in the UV to the CR geometry in the IR. At large $\xi$ and small enough $T$ the geometry has the structure depicted in Fig. 2. The radial coordinate $r$ is related to $\ell^{\prime} \hat{r}$ of the previous section by a shift that is needed to set the boundary at $r=0$. There are three regions: an AdS in the UV $(0<r \ll \ell)$, the CR geometry in the IR $(r \gg \ell)$, and an intermediate region where the linear dilaton approximation is valid $\left(\ell \ll r \ll \ell^{\prime}\right.$ with $\left.\ell^{\prime} \sim \ell \xi\right)$; the extent of the middle region grows with $\xi$, so we expect that at least some of the QNM of the CR geometry will approximate those of the full geometry when $\xi$ is large. However, in general, the quasinormal modes now acquire a nontrivial dependence on the temperature as well as on $\xi$, as we will demonstrate below.

For simplicity, instead of changing the potential, we adopt a prescription that consists in gluing the CR solution to an AdS space in the UV, so that the transition region around $r \sim \ell$ in Fig. 2 is replaced by a junction at some arbitrary point $r=r_{c}$. In this approximation and to leading order in $1 / \xi$, the spin-two correlator can again be found analytically (but the expression is too complicated to reproduce it in this article; it will be published in [8]). The modes of the completed geometry extracted from the analytic result are shown in Fig. 3 (left). They indeed depend on the temperature and approach the modes of the CR geometry only at low temperature. The cusps are related to appearance of the second set of modes at higher temperatures which we will discuss in more detail below. As the temperature grows the horizon $r_{h}$ moves to the AdS region, and the junction approximation breaks down. This happens at $T=T_{c}$ where $T_{c}=\xi / 4 \pi \ell^{\prime}$ is the temperature of the linear dilaton solution.

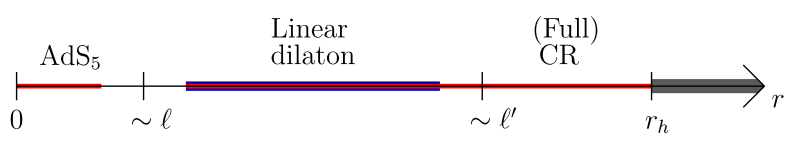

FIG. 2. The structure of the geometry for potentials asymptoting to $\mathrm{CR}$ behavior in the IR at small temperatures with large $\xi$. 

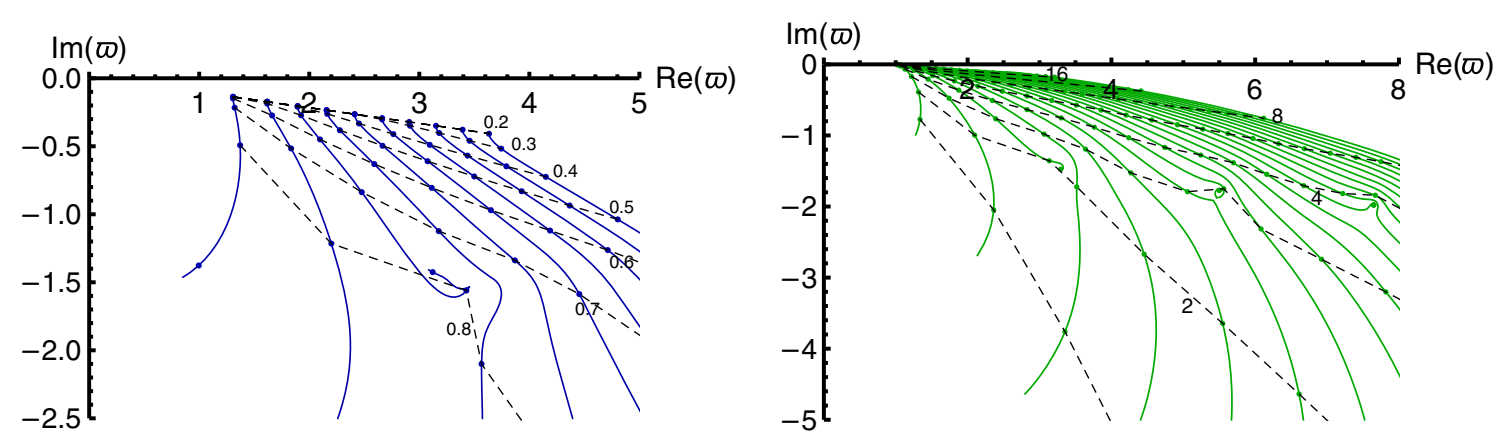

FIG. 3. The dependence of the location of quasinormal modes on the location of the horizon at $q=0$ in the setup where AdS and CR geometries were glued together. Left: The trajectories of the ten lowest QNMs on the complex $\varpi$-plane at $\xi \simeq 26.77$ as $T$ grows from $T=0.2 T_{c}$ to $T=0.91 T_{c}$. The dashed curves were added to guide the eye, they are at constant $T / T_{c}$ with values of the ratio indicated by the labels. The markers are at $T / T_{c}=0.2,0.3, \ldots 0.9$ for all curves. Right: The trajectories of the QNMs at $\xi=\infty$ on the complex $\varpi$-plane as $r_{h}$ is varied from $r_{h}=1.2 r_{c}$ (lower end points of the curves) to $r_{h}=20 r_{c}$ (upper end points). The dots are the locations of the QNMs for $r_{h} / r_{c}=\sqrt{2}, 2,2 \sqrt{2}, \ldots 16$. The dashed lines connect the locations at the same $r_{h}$ as indicated by the labels.
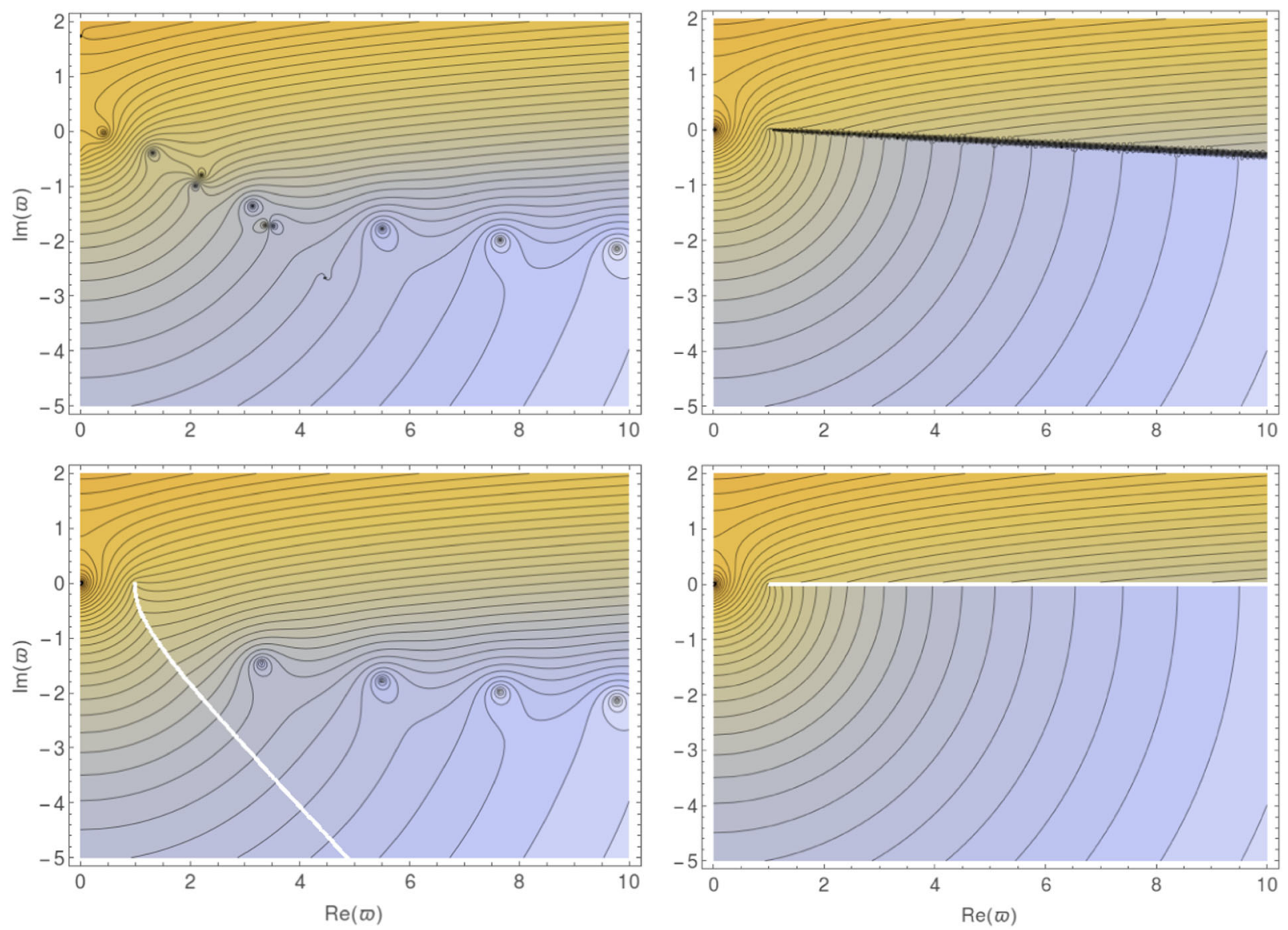

FIG. 4. The (logarithm of the) absolute value of the correlators of the energy-momentum tensor on the complex $\varpi$-plane in various approximations. The plots are for $\xi=\infty$ and $q=0$. The contours are at constant values of $\left|\tilde{G}_{\text {reg }}\right|$, with orange/yellow colors (mostly top parts of the plots) indicating small values and blue/white colors (mostly bottom parts of the plots) indicating large values. Top left: the "glued" correlator of (11) at $r_{h} / r_{c}=2$. Top right: the correlator of (11) at $r_{h} / r_{c}=20$. Bottom left: the large $\varpi$ approximation of the correlator with $r_{h} / r_{c}=2$ [8]. Bottom right: the limit of large black hole (12) of the glued correlator. 


\section{APPROACH TO CRITICALITY}

In the UV-completed geometry we can take the critical limit $\xi \rightarrow \infty$ as there is no issue with the boundary conditions. In this limit, the transverse spin-two correlator simplifies to

$$
\tilde{G}_{\mathrm{reg}}=-\frac{81 i \pi \hat{\mu}^{4}}{512 r_{c}^{4}} \frac{\hat{\mu}\left[1+e^{3 S\left(1-\frac{r_{h}}{r_{c}}\right)} \mathcal{R}\right] H_{1}^{(1)}\left(\frac{3 \hat{\mu}}{2}\right)+\left[(S-1)-e^{3 S\left(1-\frac{r_{h}}{r_{c}}\right)}(S+1) \mathcal{R}\right] H_{2}^{(1)}\left(\frac{3 \hat{\mu}}{2}\right)}{\hat{\mu}\left[1+e^{3 S\left(1-\frac{r_{h}}{r_{c}}\right)} \mathcal{R}\right] J_{1}\left(\frac{3 \hat{\mu}}{2}\right)+\left[(S-1)-e^{3 S\left(1-\frac{r_{h}}{r_{c}}\right)}(S+1) \mathcal{R}\right] J_{2}\left(\frac{3 \hat{\mu}}{2}\right)},
$$

where $\hat{\mu}=\sqrt{\varpi^{2}-q^{2}}, J_{i}$ are Bessel functions, and $H_{i}^{(1)}$ are Hankel functions of the first kind. The branch cut arising from the square root in the definition of $S$ cancels in this expression: it is invariant under $S \mapsto-S$ (which implies $\left.\mathcal{R} \mapsto \mathcal{R}^{-1}\right)$. Therefore the only singularities are poles due to the quasinormal modes.

There is a subtlety regarding the temperature in the critical limit. Namely, at any finite $\xi$ the temperature is a monotonic function of $r_{h}$, but in the critical limit the dependence of the temperature on the location of the horizon disappears and it is fixed to $T_{c}$ [4]. Our gluing procedure works when $r_{h} \gg r_{c}$.

The structure of the correlator is depicted in Fig. 4. We see that at small $r_{h}$ there are two sets of poles: one set lying on an approximately straight line, and another one for which the imaginary part is almost frequency-independent. As $r_{h}$ is increased the slope of the first set of modes decreases and eventually reaches the real axis; for this reason we identify these poles as being associated with the CR part of the geometry, whereas the region with the other poles is pushed to higher frequency and eventually these poles disappear. We do not have a good understanding of the presence of the second set of modes (having roughly constant Im $\varpi$ ), but we associate them with the AdS slice of the metric. The trajectories of the quasinormal modes as $r_{h}$ varies are shown in Fig. 3 (right). As we pointed out above the cusps in the trajectories are related to the second set of modes: as $r_{h}$ decreases some of the CR modes stop their evolution when $\operatorname{Re} \varpi \sim r_{h} / r_{c}$ and join the line of the second set of modes.

The appearance of the branch cut in the limit of large black hole can also be seen analytically by taking the limit $r_{h} \rightarrow \infty$ of (11). We obtain

$$
\tilde{G}_{\text {reg }} \rightarrow-\frac{81 i \pi \hat{\mu}^{4}}{512 r_{c}^{4}} \frac{\hat{\mu} H_{1}^{(1)}\left(\frac{3 \hat{\mu}}{2}\right)+(S-1) H_{2}^{(1)}\left(\frac{3 \hat{\mu}}{2}\right)}{\hat{\mu} J_{1}\left(\frac{3 \hat{\mu}}{2}\right)+(S-1) J_{2}\left(\frac{3 \hat{\mu}}{2}\right)} .
$$

This expression is no longer invariant under $S \mapsto-S$ and therefore we find a physical branch cut running from $\varpi=$ $\sqrt{1+q^{2}}$ to $\varpi=\infty$. This branch cut is visible in the plots on Fig. 4 (right).

\section{CONCLUSIONS}

Our results should be compared to [14-17] who also studied the dependence of the imaginary part of the lowest
QNM in systems with broken scale invariance, but found a milder dependence on the scale-breaking parameter.

It would be very interesting to understand if it is possible to make sense of the critical limit independently of the UV completion, especially in light of possible condensed matter applications, as we mentioned in the introduction. In particular we wonder if there is a generic model with infinitely many long lived excitations that we observe in our system in the critical limit $\alpha=4 / 3$.

Another interesting question is whether our result can have phenomenological applications to the QCD quarkgluon plasma. The role of the bulk viscosity, which should be important close to the deconfinement transition, has been discussed in this context (e.g. in [18]). A natural direction for further research would be to try and build a picture of how the long lived nonhydrodynamic modes affect the long-time behavior of the system.

We also observe that the critical limit $\alpha=4 / 3$ is similar to the large D limit recently discussed in [19]. We find a universal appearance of linear dilaton geometry (and its finite horizon extensions) just as in that case [20]. Finally, as discussed in [4] the black-hole solution we consider in this paper can be embedded in a higher dimensional AdS geometry where the number of internal dimensions diverge as $\alpha \rightarrow 4 / 3$.

It is also worth mentioning, even though it is not directly related to our work, that the structure of the quasinormal spectrum that we found bears a resemblance to the results of [21] who attempted to make holographic sense of the quasinormal modes of $2 \mathrm{~d}$ de Sitter space by completing it in the UV with $\mathrm{AdS}_{2}$.

Even though we studied the fluctuations at nonzero momentum, in this article we reported only on the modes at zero momentum. The behavior of the quasinormal modes as a function of $q$ will be discussed in [8].

\section{ACKNOWLEDGMENTS}

We would like to thank D. Anninos, R. Janik, E. Kiritsis, D. Mateos, and D. T. Son for discussions and helpful suggestions. This work is partially supported by the Netherlands Organisation for Scientific Research (NWO) under the VIDI Grant No. 680-47-518 and the Delta-Institute for Theoretical Physics (D-ITP) that is funded by the Dutch Ministry of Education, Culture and Science (OCW). 
[1] H. A. Chamblin and H. S. Reall, Nucl. Phys. B562, 133 (1999).

[2] U. Gürsoy and E. Kiritsis, J. High Energy Phys. 02 (2008) 032.

[3] U. Gürsoy, E. Kiritsis, and F. Nitti, J. High Energy Phys. 02 (2008) 019.

[4] U. Gürsoy, M. Järvinen, and G. Policastro, J. High Energy Phys. 01 (2016) 134.

[5] A. O. Starinets, Phys. Rev. D 66, 124013 (2002).

[6] U. Gürsoy, J. High Energy Phys. 01 (2011) 086.

[7] In order to make the statement precise, one has to modify the potential slightly.

[8] P. Betzios, U. Gürsoy, M. Järvinen, and G. Policastro (to be published).

[9] U. Gürsoy, J. High Energy Phys. 12 (2010) 062.

[10] R. Dijkgraaf, H. L. Verlinde, and E. P. Verlinde, Nucl. Phys. B371, 269 (1992).

[11] Y. Nakayama, arXiv:hep-th/0702221.

[12] The conditions $\operatorname{Re} \varpi \gtrsim \sqrt{1+q^{2}}$ for $(8)$ and $\operatorname{Re} \varpi \lesssim \sqrt{1+q^{2}}$ for (9) arise due to a saddle-point approximation required to obtain the analytic result. The actual regimes of validity for the two expressions are larger and have substantial overlap, but the conditions given in this article are enough to cover the whole complex $\varpi$-space.

[13] S. Grozdanov, N. Kaplis, and A. O. Starinets, J. High Energy Phys. 07 (2016) 151.

[14] A. Buchel, M. P. Heller, and R. C. Myers, Phys. Rev. Lett. 114, 251601 (2015).

[15] M. Attems, J. Casalderrey-Solana, D. Mateos, I. Papadimitriou, D. Santos-Olivn, C. F. Sopuerta, M. Triana, and M. Zilho, J. High Energy Phys. 10 (2016) 155.

[16] R. A. Janik, G. Plewa, H. Soltanpanahi, and M. Spalinski, Phys. Rev. D 91, 126013 (2015).

[17] T. Ishii, E. Kiritsis, and C. Rosen, J. High Energy Phys. 08 (2015) 008.

[18] S. Ryu, J.-F. Paquet, C. Shen, G. S. Denicol, B. Schenke, S. Jeon, and C. Gale, Phys. Rev. Lett. 115, 132301 (2015).

[19] R. Emparan, R. Suzuki, and K. Tanabe, J. High Energy Phys. 06 (2013) 009.

[20] R. Emparan, D. Grumiller, and K. Tanabe, Phys. Rev. Lett. 110, 251102 (2013).

[21] D. Anninos and D. M. Hofman, Classical Quantum Gravity 35, 085003 (2018). 